\title{
Zwischen Krankheitsbehandlung und Wunscherfüllung: Anti-Aging-Medizin und der Leistungsumfang solidarisch zu tragender Gesundheitsversorgung
}

\author{
Mark Schweda • Georg Marckmann
}

Eingegangen: 23. Dezember 2010 / Angenommen: 9. Mai 2011 / Online publiziert: 4. Oktober 2011

(C) Die Autor(en) 2011. Dieser Artikel ist auf Springerlink.com mit Open Access verfügbar.

Zusammenfassung Die wachsende Nachfrage nach Anti-Aging-Medizin wirft die Frage auf, welche medizinischen Leistungen ein solidarisches Gesundheitssystem tragen sollte. Die deutsche Entscheidungspraxis beruft sich auf den Begriff der Krankheit. Im Blick auf Anti-Aging wäre demnach 1) zu klären, was der Krankheitsbegriff bedeutet, 2) zu prüfen, ob das Altern sich unter diesen Begriff subsumieren lässt, um 3) abzuleiten, inwieweit Anti-Aging-Maßnahmen zur Verfügung zu stellen sind. Dieses Prozedere führt jedoch zu keinem brauchbaren Ergebnis. Unter Berufung auf den Krankheitsbegriff allein ist der Umfang solidarischer Gesundheitsversorgung nicht zu bestimmen. Allerdings lässt sich durch Reflexion auftretender Probleme ein gangbarer Entscheidungsweg aufzeigen. Er sieht eine faire Verständigung über die Frage vor, wie wir alt werden wollen.

Schlüsselwörter Anti-Aging-Medizin · Krankheitsbegriff · Altern ·

Mittelverteilung $\cdot$ Verteilungsgerechtigkeit

\section{Between therapy and wish fulfillment: anti-aging medicine and the scope of public healthcare}

Abstract Definition of the problem The increasing demand for anti-aging medicine raises the question which medical interventions should be covered within a public healthcare system. Argument The German system is centered on the concept of disease. In view of anti-aging, this could mean that we have to (1) clarify the concept of disease, (2) check

M. Schweda, M.A. $(\bowtie)$

Ethik und Geschichte der Medizin, Georg-August-Universität Göttingen,

Humboldtallee 36, 37073 Göttingen, Deutschland

E-Mail: Mark.Schweda@medizin.uni-goettingen.de

Prof. Dr. G. Marckmann

Institut für Ethik, Geschichte und Theorie der Medizin,

Ludwig-Maximilians-Universität München, Deutschland 
whether aging can be subsumed under this concept, and (3) decide to which extent, if any, anti-aging medicine should be covered. Conclusion The scope of public healthcare cannot be determined on the basis of the concept of disease alone. A reflection on the occurring problems provides reference points for a viable decision-making process involving a fair deliberation on how we want to grow old.

Keywords Anti-aging medicine $\cdot$ Concept of disease $\cdot$ Aging $\cdot$ Resource allocation $\cdot$ Distributive justice

\section{Einleitung}

Das Angebot an medizinischen Leistungen, die unter dem Label „Anti-Aging“ Beachtung und Verbreitung finden, ist im letzten Jahrzehnt förmlich explodiert. Inzwischen wird eine Vielfalt an Produkten und Verfahren zur Verfügung oder doch in Aussicht gestellt, die die Entfernung von Alterserscheinungen, die Linderung von Altersbeschwerden, die Behebung altersbedingter Funktionseinschränkungen oder gar die Verlängerung des Lebens selbst verheißen, von der Faltenbehandlung mit Botolinumtoxin über die Gabe von Hormonpräparaten zur Prävention von Osteoporose bis hin zu projektierten Eingriffen in die biologischen Mechanismen des Alterungsprozesses selbst. Und auch wenn die Möglichkeiten, Chancen und Risiken der betreffenden Ansätze oft umstritten sind, wächst die öffentliche Nachfrage doch rasant. Eine US-amerikanische Studie zur Entwicklung des weltweiten Absatzmarktes geht für 2008 von einem Marktvolumen von ca. 162,2 Mrd. \$ aus und rechnet mit einem Wachstum von $11,1 \%$ bis 2013. Besonders das ,therapeutische“ Segment, das im Rahmen der Untersuchung unterschiedslos die Behandlung von Gelenk- und Knochenzustand, Demenz, sexueller Dysfunktion, Stoffwechselstörungen sowie Herz-Kreislauf-Erkrankungen umfasst, soll bis 2013 von 66,0 auf 119,2 Mrd. \$ anwachsen [3].

Für ein Gesundheitssystem wie das deutsche, das medizinische Versorgung nicht bloß als Privatangelegenheit des Einzelnen betrachtet, sondern in gewissem Umfang als öffentliche Aufgabe begreift und aus zwangsweise zu entrichtenden Versicherungsbeiträgen finanziert, stellt diese Entwicklung eine Herausforderung dar. Denn einerseits kann die Verwendung von Mitteln im Rahmen eines in diesem Sinne „,solidarisch“ getragenen Systems nicht einfach willkürlich festgesetzt werden, sondern ist so zu begründen, dass Beteiligte und Betroffene sie im Prinzip als gerechtfertigt anerkennen können [22]. Andererseits aber stehen hinter der Nachfrage nach Anti-Aging-Maßnahmen ganz unterschiedliche Präferenzen, sodass über ihre Beurteilung selbst bei unterstellter Wirksamkeit der gewünschten Produkte und Dienstleistungen nicht leicht Einigkeit zu erzielen ist: Handelt es sich um übersteigerten Jugendwahn, wenn ein 60-jähriger Freizeitsportler seine Kniegelenke mit Hyaluronsäurepräparaten aufspritzen lässt, um weiter eine gute Figur auf dem Surfboard zu machen? Hat die 67-jährige Akademikerin, die zum Erhalt ihrer intellektuellen Leistungsfähigkeit Nootropika einsetzt, nur ein Luxusproblem? Ist es anstößig, wenn der vitale 70-Jährige auf Viagra setzt, um auch künftig ein sexuell erfülltes Leben führen zu können?

Wie die Beispiele deutlich machen, berührt die Frage nach dem Umgang mit AntiAging innerhalb eines solidarischen Gesundheitssystems die normativen Prinzipien, auf deren Grundlage dieses System operiert: Welche Maßnahmen sollte der Leistungskatalog der Gesetzlichen Krankenversicherung (GKV) umfassen und welche nicht? Was begründet überhaupt einen Anspruch auf medizinische Leistungen bzw. die entsprechende Verpflichtung zu ihrer Bereitstellung? Diese Fragen gewinnen im Fall des Anti-Aging noch dadurch an Brisanz, dass viele der fraglichen Produkte und Dienstleistungen ausgesprochen kost- 
spielig sind. Ihre Aufnahme dürfte eine beträchtliche Belastung für das öffentliche Gesundheitssystem darstellen, ihre Ausgrenzung indes zu erheblichen sozialen Verwerfungen führen [15].

Hält man sich an geltendes Recht, so scheinen die genannten Fragen freilich relativ einfach zu beantworten zu sein. Die erklärte Aufgabe der GKV ist es, „die Gesundheit der Versicherten zu erhalten, wiederherzustellen oder ihren Gesundheitszustand zu bessern.“ (Sozialgesetzbuch (SGB) V, § 1,1) Da die Begriffe „Gesundheit“ und „Krankheit“ dabei eine vollständige Dichotomie bilden, besteht entsprechend ein Anspruch auf medizinische Versorgung, ,wenn sie notwendig ist, um eine Krankheit zu erkennen, zu heilen, ihre Verschlimmerung zu verhüten oder Krankheitsbeschwerden zu lindern“ (SGB V, § 27). Auch die sozialrechtlichen Kriterien der Wirksamkeit, Notwendigkeit, Wirtschaftlichkeit und des Nutzens (SGB V, §§ 2, 12 und 135) sind letztlich auf diesen Zweck bezogen. Demnach wäre im Blick auf die Anti-Aging-Medizin 1) zu klären, was der Krankheitsbegriff bedeutet, 2) zu prüfen, ob das Altern bzw. die jeweils anvisierten Alterserscheinungen sich unter diesen Begriff subsumieren lassen, um 3) aus 1) und 2) eine Antwort auf die Frage abzuleiten, inwieweit wirksame Anti-Aging-Maßnahmen im Rahmen öffentlicher Gesundheitsversorgung zur Verfügung zu stellen sind.

Wie sich im Folgenden zeigen wird, führt dieser Weg jedoch zu keinem brauchbaren Ergebnis. Auf jeder der drei Ebenen treten Probleme auf, die eine Lösung am Ende in weite Ferne rücken lassen. In diesem Sinne ist das Ergebnis zunächst ein negatives: Unter Berufung auf den Krankheitsbegriff allein ist der Umfang solidarischer Gesundheitsversorgung nicht zu bestimmen, weder im Blick auf Anti-Aging noch im Allgemeinen. Allerdings lassen sich durch Reflexion der auftretenden Probleme immerhin Anhaltspunkte für einen gangbaren Entscheidungsweg gewinnen, der abschließend kurz skizziert werden soll.

\section{Sozialrechtlicher Krankheitsbegriff und medizintheoretische Diskussion}

Laut Sozialgesetzbuch V ist der Begriff der Krankheit für die Entscheidung über sozialrechtliche Leistungsansprüche zentral. Dennoch liegt dem sozialrechtlichen Krankheitsbegriff kein klar definiertes Konzept zu Grunde, dessen Merkmale sich ohne Weiteres als Kriterien für die Umfangsbestimmung medizinischer Versorgung - oder gar zur Bewertung einzelner Ansprüche - heranziehen ließen. Es handelt sich vielmehr um einen unbestimmten Rechtsbegriff, dessen Konkretisierung im Verlauf der Rechtsprechung selbst erfolgt. Einschlägig ist die vom Bundessozialgericht geprägte Auslegungsformel, durch die Krankheit als ein „regelwidriger Körper- oder Geisteszustand“ bestimmt wird, „der ärztlicher Behandlung bedarf und/oder Arbeitsunfähigkeit zur Folge hat" (Entscheidungen des Bundessozialgerichts (BSGE) 35, 10, 12). Ersichtlich hat das Gericht sich bemüht, Anhaltspunkte namhaft zu machen, anhand deren im Einzelfall über das Vorliegen einer Krankheit und damit die Berechtigung eines Leistungsanspruchs entschieden werden kann. Bei näherer Betrachtung zeigt sich aber, dass jedes der genannten Teilkriterien selbst wiederum mit Schwierigkeiten behaftet ist [37].

Das Kriterium der Behandlungsbedürftigkeit knüpft den Krankheitsbegriff an die Voraussetzung, dass der betreffende Zustand ,ohne ärztliche Hilfe nicht mit Aussicht auf Erfolg behoben, mindestens aber gebessert oder vor Verschlimmerung bewahrt werden kann oder wenn ärztliche Behandlung erforderlich ist, um Schmerzen oder sonstige Beschwerden zu lindern“ (BSGE 35, 10, 12). Freilich ist die Auskunft, dass genau diejenigen Zustände einen Anspruch auf ärztliche Behandlung begründen, die dieser Behandlung bedürfen, nahezu inhaltsleer und hat ohne Spezifikation der fraglichen Zustände kaum Substantiel- 
les zur Bewertung sozialrechtlicher Leistungsansprüche beizutragen. Auch der Verweis auf medizinische Indikationen hilft nicht weiter, sondern schiebt die Frage nur den Ärzten zu, unter denen in diesem Punkt ebenfalls kein Einvernehmen herrscht. Gerade angesichts der wachsenden Nachfrage nach Anti-Aging wird innerhalb der Profession kontrovers diskutiert, ob z. B. das Absinken des Östrogenspiegels im Klimakterium oder das Nachlassen der Gedächtnisleistung im höheren Lebensalter wirklich ärztlicher Behandlung bedürfen [25].

Das Kriterium der Arbeitsunfähigkeit bezieht sich auf einen ,durch Krankheit oder Unfall hervorgerufene[n] Körper- und Geisteszustand, aufgrund dessen der Versicherte seine bisherige Erwerbstätigkeit überhaupt nicht oder nur unter der in absehbar nächster Zeit zu erwartenden Gefahr der Verschlimmerung seines Zustandes weiter ausüben kann" (BSGE 19, 179). Freilich bezeichnet auch dieser Begriff der Arbeitsunfähigkeit keine objektive Größe. Seine Bedeutung ist von historisch und kulturell variablen Kontextbedingungen abhängig. Zu ihnen zählen die Form und das spezifische Anforderungsprofil der Arbeiten, die die gesellschaftliche Praxis bestimmen. Auch im Blick auf das Individuum ist der Begriff nicht objektiv, etwa im Sinne einer physikalischen Belastbarkeitsgrenze zu verstehen, sondern zielt auf die Zumutbarkeit der fraglichen Arbeit ab und schließt insofern moralische Maßstäbe ein [36]. Es war nicht zuletzt die Ende des 19. Jahrhunderts einsetzende Entwicklung des Sozialversicherungssystems selbst, in deren Verlauf solche Standards etabliert und immer wieder auch verschoben wurden. Gerade im Blick auf das Altern hatte dieser Vorgang freilich ambivalente Konsequenzen: So führte die Einführung einer Gesetzlichen Rentenversicherung zwar einerseits dazu, dass man nicht mehr schuften muss, bis man - im wahrsten Sinne des Wortes - umfällt, sondern sich ab einem politisch festgesetzten Renteneintrittsalter der Arbeitswelt und ihren Leistungs- und Effizienzkriterien entziehen kann. Andererseits aber verliert Arbeitsunfähigkeit damit im Blick auf Personen jenseits dieser Altersgrenze jede sozialrechtliche Relevanz. Innerhalb einer Gruppe, die geradezu durch pauschal unterstellte Arbeitsunfähigkeit definiert ist, taugt diese kaum zur anspruchsbegründenden Unterscheidung von Krankheit und Gesundheit. Zudem wird argumentiert, dass die Medizin gerade dem Erhalt der Arbeitsfähigkeit im Alter dienen und so zur Entlastung der Sozialen Sicherungssyteme beitragen könnte [31].

Grundlegend für den sozialrechtlichen Krankheitsbegriff ist das Kriterium der Regelwidrigkeit. Dass Krankheit etwas mit einer Irregularität, einer Normabweichung zu tun hat, erscheint auch im alltagspraktischen Zusammenhang einleuchtend. Bei näherer Betrachtung stellt sich allerdings die Frage, welche Art von Norm zu Grunde gelegt wird, wenn von einer Abweichung die Rede ist. Die nahe liegende Annahme, es müsste sich um eine statistische Norm handeln, eine Art Durchschnittswert, hält der Überprüfung jedenfalls nicht stand. Denn zum einen gibt es vielfältige Erscheinungen, die als Normabweichungen in diesem statistischen Sinne gelten müssen, ohne dass wir ihnen deshalb einen Krankheitswert zuschreiben würden, z. B. eine seltene Blutgruppe. Und zum anderen lassen sich verbreitete, statistisch normale Erscheinungen benennen, die wir gleichwohl als krankhaft bezeichnen, z. B. Karies oder Arteriosklerose. Auch Alterserscheinungen werden ungeachtet statistischer Prävalenz unter Menschen des betreffenden Lebensalters durchaus als Krankheiten anerkannt. So hat das Bundessozialgericht entschieden, „dass als Krankheit im Sinne der gesetzlichen Krankenversicherung solche Regelwidrigkeiten nicht ausgeschlossen sind, die auf einen Alterungsprozess zurückzuführen sind“ (Bundessozialgericht (BSG) Az: B 8 KN 9/98 KR R). Entsprechend werden Hörhilfen und Zahnersatz für ältere Menschen zumindest öffentlich bezuschusst. Tatsächlich wird das Kriterium der Regelwidrigkeit in der Rechtsprechung durch die Idee einer „Abweichung vom Leitbild des gesunden Menschen“ (BSGE 26, 240) spezifiziert und damit ausdrücklich nicht auf statistische Durchschnittswerte, sondern auf gesellschaftliche Idealvorstellungen bezogen. Freilich sind solche Ideale nicht nur histo- 
risch wandelbar, sondern treten in modernen, pluralistischen Gesellschaften auch stets im Plural auf [12], sodass auch die Berufung auf einen durch „Konsens und Plausibilität“ ([14], S. 18) qualifizierten Krankheitsbegriff nur noch bedingt weiterhilft. Schließlich erweitert gerade der medizinische Fortschritt unablässig den Spielraum des Möglichen und erzeugt so immer neue Wünsche und Begehrlichkeiten gegenüber Medizin und Gesundheitswesen, sodass die Bezugnahme auf gesellschaftliche Leitbilder untauglich zur Begrenzung von Leistungsansprüchen sein dürfte [27].

Angesichts dieser Unschärfen scheint es nahe zu liegen, den sozialrechtlichen Krankheitsbegriff zum Zweck der Umfangsbestimmung der GKV mit Hilfe medizintheoretischer Überlegungen zu präzisieren. Allerdings sieht man sich dabei mit einer großen Bandbreite theoretischer Ansätze und einer entsprechenden Vielfalt unterschiedlicher Krankheitsbegriffe konfrontiert [28]. Im Hinblick auf die Deutung des grundlegenden Gedankens der Normabweichung lassen sich dennoch - grob vereinfachend - zwei Grundpositionen ausmachen: Naturalistische Ansätze betrachten die für den Krankheitsbegriff maßgebliche Norm als eine Art biologischer Gesetzmäßigkeit und sehen Krankheit daher als eine objektiv feststellbare Tatsache an. Normativistische Ansätze deuten sie dagegen im Sinne individueller oder gesellschaftlicher Wertmaßstäbe, sodass Krankheitszuschreibungen für sie stets gewisse Wertungen implizieren (dazu ausführlich: [36]).

Ein prominentes Beispiel für einen naturalistischen Krankheitsbegriff ist die biostatistische Konzeption von Boorse, die von der Vorstellung eines „,normal species functioning“ ([5], S. 567) ausgeht. Ihr zufolge ist Krankheit als Abweichung eines Organismus von der statistisch normalen Funktionsfähigkeit bei Organismen einer entsprechenden Referenzklasse definiert. Damit wird einerseits dem Gesichtspunkt der statistischen Abweichung Rechnung getragen. Andererseits wird der Bezugspunkt des Vergleichs im Sinne der Funktionsfähigkeit des betreffenden Organismus spezifiziert, wobei Boorse unter einer Funktion den Beitrag eines Organs oder Prozesses zu Überleben und Fortpflanzung des Individuums versteht. Nicht jede Abweichung eines Organismus von einer statistischen Norm ist also per se schon als Krankheit zu bezeichnen, sondern nur diejenige, die seine Fähigkeit zur Selbsterhaltung und Reproduktion im Verhältnis zu anderen Individuen seiner Art herabsetzt. Dieser Ansatz mag geeignet erscheinen, dem sozialrechtlichen Kriterium der Regelwidrigkeit einen klaren Sinn zu verleihen. Im Blick auf die Frage der Umfangsbestimmung öffentlicher Gesundheitsversorgung erweist er sich dennoch als unbrauchbar, und zwar gerade hinsichtlich des Anti-Aging. Denn zunächst muss als zweifelhaft gelten, ob überhaupt auf rein naturalistischer Basis zur Bestimmung von Funktionen und zur Bildung von Referenzklassen zu gelangen ist [36]. Von einer Funktion kann doch offenbar immer nur im Hinblick auf ein Ziel gesprochen werden, wobei äußerst umstritten ist, ob solche Ziele der Natur selbst innewohnen oder ihr von einem Beobachter unterstellt werden. Und auch Referenzklassen sind keine Klassen, in die die Natur von sich aus zerfällt, sondern setzen Unterscheidungen voraus, die nur von einem Beobachterstandpunkt aus zu treffen sind. Indem Boorse selbst etwa Überleben und Fortpflanzung als maßgebliche Ziele unterstellt und seine Referenzklassen zudem geschlechts- und altersspezifisch fasst, scheinen in seinen vermeintlich wertneutralen Krankheitsbegriff wertende Vorstellungen bezüglich Altern und Alter einzugehen.

Normativistische Konzeptionen haben demgegenüber den Vorzug, die normativen Implikationen des Krankheitsbegriffs offen thematisieren zu können. Allerdings hängt die Aussagekraft der verschiedenen Ansätze davon ab, wie sie die fraglichen Bewertungsmaßstäbe interpretieren. Als unzulänglich dürfte sich etwa ein radikal subjektivistischer Krankheitsbegriff erweisen, der alle individuell unerwünschten Zustände - oder doch diejenigen, die den „maßgeblichen Zielen“ ([30], S. 22) der Betroffenen entgegen stehen - als Krankheit zu fassen erlaubt. Denn damit würde das Urteil über Gesundheit und Krankheit von den 
Präferenzen der Einzelnen abhängig gemacht und der Anspruch auf medizinische Versorgung so letztlich in ihr subjektives Belieben gestellt. Die faktische Nachfrage würde zum anspruchsbegründenden Prinzip. Wie gerade die eingangs skizzierte Nachfrageentwicklung im Bereich Anti-Aging zeigt, würde so der finanzielle Spielraum des öffentlichen Gesundheitswesens bald gesprengt. Den zusehends diversifizierten und expandierenden Ansprüchen könnten keine legitimen Grenzen mehr gezogen werden. Um eine solche Entwicklung zu vermeiden, müsste der verwendete Krankheitsbegriff sich auf Normen stützen können, die auf allgemeine Zustimmung rechnen dürfen. Tatsächlich gibt es Ansätze, solche Normen etwa in der menschlichen Natur zu verankern [18] oder aus universell gültigen moralischen Prinzipien abzuleiten [4]. Freilich scheint zweifelhaft, ob dieser Weg zu einer tragfähigen Lösung führt. Immerhin müssten die fraglichen Normen einerseits gehaltvoll genug sein, um konkrete Schlussfolgerungen für den Leistungskatalog der GKV zu erlauben, andererseits aber doch zugleich so zu begründen sein, dass sie allgemein anerkannt werden können. $\mathrm{Zu}$ Recht erklären Befürworter des Anti-Aging, es sei nicht akzeptabel, der Allgemeinheit die betreffenden Maßnahmen unter Berufung auf private Wertvorstellungen und religiöse Welt- und Menschenbilder vorzuenthalten [24].

Somit erweisen sich auch Versuche, den sozialrechtlichen Krankheitsbegriff mit Hilfe medizintheoretischer Überlegungen zu unterfüttern, als nicht zielführend. Statt dem Kriterium der Regelwidrigkeit einen klaren Sinn zu verleihen und uns so einer konkreten Antwort auf die Frage nach dem Umgang mit der Anti-Aging-Medizin näher zu bringen, verweisen sie auf das grundlegende Problem, wie die normativen Implikationen des Krankheitsbegriffs zu deuten und zu begründen sind. Immerhin scheint der Bezug auf den Krankheitsbegriff geeignet zu sein, zumindest diejenigen Anti-Aging-Verfahren aus dem Leistungskatalog auszugrenzen, die erklärtermaßen gar nicht die Behandlung von Krankheiten zum Ziel haben. Das betrifft zum einen kosmetische oder Wellness-Maßnahmen, also etwa die Faltenbehandlung mit Botox oder die Kur im „Anti-Aging-Spa“. In ihrem Fall greift ohnehin eine Änderung des Sozialgesetzbuchs, nach der Maßnahmen von der Kostenübernahme ,,ausgeschlossen“ sind, „,bei deren Anwendung eine Erhöhung der Lebensqualität im Vordergrund steht" (SGB V, § 34). Auf der anderen Seite fallen auch alle Maßnahmen heraus, die auf eine radikale Lebensverlängerung, die Ausweitung der Lebensspanne um Jahrzehnte oder Jahrhunderte abzielen. Übrig bleibt damit indes just der Kernbereich jener Maßnahmen, deren Einstufung strittig ist, weil sie in der Grauzone zwischen Krankheitsbehandlung und Wunscherfüllung operieren. Sie berühren die Frage nach der Abgrenzung von Altern und Krankheit bzw. normalen, physiologischen Alterungsprozessen und pathologischen Entwicklungen.

\section{Altern zwischen physiologischem Prozess und pathologischem Phänomen}

Der eingangs skizzierte sozialrechtliche Entscheidungsweg führt zu der Erörterung der Frage, ob es sich beim Altern um eine Krankheit handelt. Bezogen sich die vorangehenden Überlegungen auf den Sinngehalt des Krankheitsbegriffs, so ist hier sein Umfang tangiert: Fällt das Altern unter den Begriff der Krankheit? Weist es zumindest einige der Merkmale auf, die diesen Begriff definieren? In Anbetracht des ergebnislosen Ausgangs des letzten Abschnitts liegt es freilich nahe, die Argumentation bereits an dieser Stelle abzubrechen: Wenn nicht einmal definitiv gesagt werden kann, was überhaupt unter einer Krankheit zu verstehen ist, scheint sich die Frage zu erübrigen, ob es sich bei einem bestimmten Phänomen wie dem Altern um eine Krankheit handelt - zumal der Begriff des Alterns selbst ebenfalls keineswegs eindeutig festgeschrieben ist [17]. 
Gleichwohl lassen sich auf der Grundlage der eingeführten Unterscheidung von Krankheitsbegriffen immerhin verschiedene denkbare Verhältnisbestimmungen von Krankheit und Altern skizzieren und in ihren Konsequenzen für den Leistungsumfang der GKV hypothetisch durchspielen. Dazu sind auch im Hinblick auf die Debatte um die Beziehung von Krankheit und Altern - grob vereinfachend - zwei Grundpositionen auseinanderzuhalten: Die erste geht von einer vollständigen Dichotomie zwischen Altern und Krankheit aus, die zweite von einem Kontinuum zwischen beidem [8]. Vertreter der Dichotomiethese sind der Ansicht, dass Krankheit und Altern zwei verschiedene Prozesse sind. Zwar mag es gewisse Erkrankungen geben, die gewöhnlich mit dem Altern einhergehen. Doch diese sind grundsätzlich vom natürlichen, ,physiologischen“ Alterungsprozess zu unterscheiden. Die Anhänger der Kontinuitätsthese wenden ein, dass sich eine solche Unterscheidung kaum trennscharf treffen lässt. In Wahrheit sei das Altern selbst nichts anderes als die Summe aller über den Verlauf des Lebens akkumulierten Erkrankungen und Schädigungen, die zuletzt zum Tode führen.

Obwohl historisch beide Standpunkte vertreten wurden, verzeichnen Sozialwissenschaftler einen Trend zur Pathologisierung - und damit zu einer an der Kontinuitätsthese orientierten Auffassung - des Alterns [33]. Zur Illustration wird oft auf das Verschwinden der „Altersschwäche“ aus den Todesursachenstatistiken verwiesen. Hatte man einst angenommen, dass das Versiegen der Lebenskräfte im Laufe des Alterungsprozesses dem naturgemäßen Gang der Dinge entspreche, an dessen Schlusspunkt ein „natürlicher Tod“ durch „Auszehrung“ oder „Entkräftung“ stehe, so erschien diese Sicht unter dem wachsenden Einfluss naturwissenschaftlicher Erklärungsmodelle und technischer Eingriffsmöglichkeiten immer weniger zufriedenstellend. Die mit dem Alter auftretenden, zuletzt zum Tode führenden Prozesse sind nun ätiologisch aufzuschlüsseln und kausal zu behandeln. Es scheint in der Konsequenz dieses Ansatzes zu liegen, dass sich mit der Ausschaltung aller Alterskrankheiten zuletzt das, was wir heute noch „Altern“ nennen und als natürlichen Prozess auffassen, selbst verflüchtigen wird.

Kombiniert man die Unterscheidung der Krankheitsbegriffe mit der der Verhältnisbestimmungen von Krankheit und Altern, ergeben sich vier idealtypische Positionen. So lässt sich die traditionelle medizinische Standardauffassung des Alterns als Dichotomiekonzeption auf Basis eines naturalistischen Krankheitsbegriffs rekonstruieren. Demnach ist das Altern ein allen Organismen inhärenter, natürlicher Vorgang und nicht ein Komplex pathologischer Erscheinungen. Die physiologische Alterung mag regelmäßig mit gewissen Erkrankungen einhergehen oder sogar zu ihnen disponieren, ist jedoch grundsätzlich von ihnen zu unterscheiden [23]. Dies ist erkennbar auch die Vorstellung, auf die der oben skizzierte biostatistische Krankheitsbegriff hinausläuft. Schließlich bestimmt Boorse die „normale speziestypische Funktionsfähigkeit“ im Verhältnis zu altersspezifischen Referenzklassen und setzt so bereits im Theoriedesign einen Rückgang der Funktionsfähigkeit über den Lebensverlauf voraus. Beeinträchtigungen körperlicher und geistiger Funktionen können in diesem Rahmen prinzipiell nicht als pathologische Erscheinungen interpretiert werden, solange sie dem Durchschnitt der jeweiligen Altersgruppe entsprechen. Ein ähnlicher Gedanke prägt auch den in der Geriatrie vorherrschenden altersmedizinischen Ansatz, der von ,physiologische[n] Altersveränderungen auf der Ebene des Gesamtorganismus, der einzelner Organsysteme und auf zellulär-molekularer Ebene“ ausgeht und daraus folgert, dass im Alter bei der Diagnose und Behandlung von Erkrankungen entsprechend angepasste Standards in Anschlag zu bringen sind: „Normwerte, z. B. für Lungenfunktions-, HerzKreislauf- oder neurophysiologische Parameter ändern sich mit zunehmendem Lebensalter. Die Kenntnis dieser Veränderungen ist zur Beurteilung und Differenzierung zwischen normal und pathologisch unerläßlich“"([9], S. 5). Für die Frage des Leistungsumfangs scheinen 
sich aus einer solchen Konzeption restriktive Konsequenzen zu ergeben: Wenn man von der Vorstellung eines in der Natur des Organischen angelegten, von Krankheiten zu unterscheidenden Abbau- und Verfallsprozesses und einer biologisch begrenzten Lebensspanne des Menschen ausgeht, scheint der medizinische Kampf gegen das Altern nicht angebracht zu sein. Nicht nur der Ausschluss des Anti-Aging aus der Gesundheitsversorgung, sondern auch die Begrenzung medizinischer Leistungen auf Grund des Lebensalters könnte auf dieser Grundlage gerechtfertigt werden [10].

Caplan [11] legt den Finger in die Wunde der naturalistischen Dichotomiekonzeption, wenn er fragt, wieso wir das Altern im Unterschied zu Krankheiten als normalen oder natürlichen Prozess hinnehmen, obwohl es doch offenkundig ebenfalls die Morbidität von Organismen erhöht und zuletzt zum Tode führt, ohne eine ersichtliche biologische Funktion zu haben. Mit der faktischen Allgegenwart und Vertrautheit der das chronologische Altern begleitenden Seneszenzprozesse lasse sich eine solche Sichtweise allenfalls erklären, aber nicht rechtfertigen. Schließlich gebe es nicht nur vergleichbar verbreitete Krankheiten; es sei auch eine Welt denkbar, in der Organismen nicht altern. Tatsächlich erweise sich das Altern im Zuge des medizinischen Fortschritts bereits als veränderliche Erscheinung, die mit den Mitteln moderner Medizin beeinflusst werden kann. Altersspezifische Normwerte bei der Diagnose und Behandlung von Krankheiten zu Grunde zu legen, erscheint vor diesem Hintergrund wie die willkürliche Festschreibung eines historisch kontingenten Status Quo [20]. So entfaltet Caplan in Auseinandersetzung mit dem impliziten Traditionalismus der Dichotomiethese eine naturalistische Kontinuitätskonzeption. Das Altern erscheint als pathologischer Vorgang, der medizinisch zu behandeln ist, ähnlich wie sich schon andere vermeintliche Naturgegebenheiten im Lichte moderner Medizin als behebbare Krankheiten entpuppt haben. Freilich geht dieser Versuch einer Revision der Standardauffassung selbst ebenfalls recht unbedarft mit dem schillernden Begriff der Natürlichkeit um [34]. Vor allem aber streift sein Verweis auf die gesellschaftliche Sicht und historische Wandlung des Krankheitsbegriffs bereits die Grenze des naturalistischen Paradigmas.

In der Perspektive des normativistischen Ansatzes verweist die Erkundigung nach der Beziehung von Krankheit und Altern auf die Frage, inwiefern das Altern und seine Begleiterscheinungen negativ zu bewerten sind. Deshalb müssen Vertreter einer Kontinuitätsthese auf normativistischer Grundlage das Bild des Alterns so düster zeichnen bzw. die normativen Ansprüche derart hoch hängen, dass sich die für ihren Krankheitsbegriff maßgebliche negative Bewertung ergibt. Tatsächlich werden insbesondere Anhänger des Anti-Aging nicht müde zu betonen, dass das Altern zu den größten Übeln des menschlichen Daseins zählt und unsere innigsten Wünsche und zentralsten Projekte zunichte macht [6]. Regelmäßig wird der alte Menschheitstraum von ewiger Jugend und Schönheit zur Legitimation des Unternehmens zitiert. Ohnehin mache es die Würde des Menschen aus, vermeintlich naturgegebene Grenzen zu überwinden und sich selbst zu vervollkommnen [7]. Bisweilen wird Langlebigkeit geradezu zu einem fundamentalen Menschenrecht erklärt [19]. So könnten sich Vertreter einer normativistischen Kontinuitätsthese durchaus im Ideenreservoir des Anti-Aging bedienen. Freilich ist dieses auch selbst an der Verschiebung der normativen Maßstäbe beteiligt, in deren Licht das Altern bewertet wird, und leistet so der Pathologisierung, auf die es zunächst nur zu reagieren scheint, zugleich aktiv Vorschub.

Wollte man gegenüber dieser Position eine normativistische Dichotomiethese vertreten, so müsste man plausibel machen, dass das Altern etwas Wertvolles darstellt - sei es trotz oder gerade wegen der mit ihm verbundenen Beeinträchtigungen. Auf der Basis individueller Präferenzen ließe sich etwa im Sinne eines Nutzenkalküls argumentieren, dass unerwünschte Alterserscheinungen unlösbar mit gewissen erwünschten Erscheinungen verknüpft sind, die sie mindestens aufwiegen, etwa Formen der Persönlichkeitsentwicklung 
wie die Ausbildung von Weisheit oder soziale Vorteile wie zwischenmenschliche Fürsorge. Der erste Fall weist insofern bereits über eine präferenzorientierte Position hinaus, als er die Vorstellung eines mit dem Altern einhergehenden Reifungsprozesses und damit eine Vision guten Lebens ins Spiel bringt, zu der das Alter wesentlich gehört. Vertreter einer solchen Auffassung erklären etwa, dass sich wesentliche Züge des Menschen erst im Alter voll entfalten [32] oder dass das Leben nur unter den Bedingungen von Endlichkeit und Vergänglichkeit einen Sinn haben kann [21]. Freilich beruhen solche „Kontingenzargumente“ [2] meist auf metaphysischen oder religiösen Prämissen, die in modernen, pluralistischen Gemeinwesen nicht zur Grundlage allgemein bindender sozialpolitischer Regelungen gemacht werden können.

So zeigt sich, dass auch die Beziehung von Krankheit und Altern nicht ohne Weiteres zu spezifizieren ist, selbst wenn man von einer bestimmten Krankheitskonzeption ausgeht. Ob wir das Altern als Krankheit bezeichnen, hängt nicht nur vom zu Grunde gelegten Krankheitsbegriff, sondern auch von der vorausgesetzten Auffassung des Alterns selbst ab. Hier eröffnet sich ein breites, normativ aufgeladenes Spektrum: Ob Altern als natürliche Entwicklung angesehen wird, die es hinzunehmen gilt, oder gar als Vollendung des menschlichen Lebens - oder ob es als pathologischer Prozess und Geißel der Menschheit betrachtet wird und mit allen Mitteln moderner Medizin bekämpft werden soll, lässt sich nicht objektiv, quasi auf naturwissenschaftlicher Basis entscheiden. Es hängt von sozio-kulturell geprägten und historisch wandelbaren Auffassungen des Menschen und des guten Lebens ab.

\section{Krankheitsbegriff und Mittelverteilung}

Das Ergebnis der ersten Abschnitte ist negativ. Statt einer Antwort auf die Ausgangsfrage ergibt sich nur eine Kaskade gleichermaßen problematischer Argumentationslinien. So fragt sich, ob es überhaupt sinnvoll ist, den sozialrechtlich vorgezeichneten krankheitsbasierten Entscheidungsweg weiter zu verfolgen. Gewiss sind die bisher vorgebrachten Einwände keineswegs schlagend. Sie markieren Probleme, die vielleicht im Zuge verstärkter Bemühungen um eine angemessene Begrifflichkeit behoben werden könnten. Womöglich muss man einfach noch eingehender über den Krankheitsbegriff und das Verhältnis von Altern und Krankheit nachdenken, um zu einer tragfähigen Lösung zu gelangen.

Hier sind indes Zweifel angebracht. Selbst wenn der Krankheitsbegriff sich eindeutig definieren ließe und das Altern tatsächlich als Krankheit in diesem Sinne zu bezeichnen wäre, scheint daraus unmittelbar nichts für den Umgang mit der Anti-Aging-Medizin zu folgen. Denn aus der bloßen Feststellung einer Tatsache lässt sich ohne normative Zusatzprämissen keine normative Schlussfolgerung ziehen. Dass sich eine Person in einem - wie auch immer zu definierenden - Zustand befindet, den wir „Krankheit“ nennen, mag uns psychologisch dazu motivieren, ihr Hilfe zuteil werden zu lassen. Es vermag aber logisch noch keinen Anspruch auf medizinische Versorgung zu begründen. Dies zu behaupten käme einem naturalistischen Fehlschluss gleich, dem logisch unzulässigen Übergang von Seinszu Sollensaussagen. Weder aus der Subsumption des Alterns unter einen naturalistisch gefassten noch aus der unter einen normativistisch formulierten Krankheitsbegriff lässt sich daher ohne Weiteres eine Orientierung für den Umgang mit dem Anti-Aging gewinnen.

Besonders deutlich wird dieses Problem an naturalistischen Dichotomiekonzeptionen. Selbst wenn man davon ausgeht, dass der Begriff der Krankheit sich auf naturalistischer Basis definieren lässt, erwiese sich gerade ein solcher „wertfreier“ Krankheitsbegriff als normativ unergiebig. Zwar mag sich die von Boorse vorausgesetzte Altersrelativität normaler Funktionsfähigkeit unter rein deskriptiven Gesichtspunkten mit Verweis auf die faktische 
statistische Altersverteilung gesundheitlicher Funktionseinschränkungen plausibel machen lassen. Ein solcher Verweis auf statistische Normalität ist jedoch nicht dazu geeignet, eine bestimmte präskriptive Normierung öffentlicher Gesundheitsversorgung zu rechtfertigen. Wenn diese Normierung überdies auf die Begrenzung von Ansprüchen auf alternsbezogene Maßnahmen hinausliefe, die gerade darauf abzielen, die statistische Ausgangslage zu verändern, erschiene das Vorgehen geradezu zynisch: die normative Festschreibung des gegebenen Istzustandes. Dies ist genau die Stoßrichtung von Caplans Kritik am Traditionalismus naturalistischer Dichotomiekonzeptionen, der von Anti-Aging-Protagonisten auch als geriatrischer ,cult of death“ bezeichnet wird. Würde man freilich unterschiedliche Altersstufen bei der Definition sozialrechtlicher Gesundheitsstandards überhaupt nicht berücksichtigen, wie es Vertreter naturalistischer Kontinuitätsthesen fordern, käme dies sozialrechtlich im Effekt einer radikalen Entgrenzung des Anspruchs auf medizinische Versorgung gleich. Alle medizinischen Maßnahmen, die ein altersbedingtes Absinken des individuellen Gesundheitszustands unter den gesamtgesellschaftlichen Durchschnitt verhindern könnten, wären solidarisch zu tragen. Damit würde das mittlere Lebensalter faktisch zum gesundheitlichen Normalfall erklärt und so womöglich eine Tendenz zur Pathologisierung und Medikalisierung des Alterns eingeleitet ([13], S. 162). Begründungslogisch gesehen wäre allerdings nichts bewiesen, denn ob es sich beim Altern tatsächlich um einen abweichenden Prozess handelt, der medizinisch behandelt werden sollte, ist eine genuin normative Frage.

Doch auch auf Seiten der normativistischen Konzeptionen sieht es kaum besser aus: Zunächst kann eine Umfangsbestimmung solidarischer Gesundheitsversorgung, die sich auf individuelle oder gesellschaftliche Werte beruft, diese weder aus der Luft greifen noch einfach theoretisch konstruieren. Sie hat von den tatsächlichen Wertvorstellungen der betreffenden Personen und Gruppen auszugehen und diese zu diesem Zweck allererst empirisch zu erheben. Natürlich gibt es eine Fülle sozialwissenschaftlicher Untersuchungen, die empirische Informationen zu Vorstellungen vom und Einstellungen zum Altern liefern [1]. Allerdings bleibt dabei das Problem der Legitimation bestehen. Ob das Altern nun von den Befragten als Gut gefeiert oder als Übel verachtet wird: Dass Einzelne oder Gruppen de facto gewisse Präferenzen haben, heißt noch nicht, dass ihre Ansprüche allgemein akzeptabel sind. Schließlich kommt es für die Gültigkeit einer Überzeugung nicht auf die Zahl der Personen an, die sie vertreten, sondern auf die Anerkennungsfähigkeit der sie tragenden Argumente.

Selbst wenn sich alle begrifflichen Unklarheiten ausräumen ließen, würde der verfolgte Entscheidungsweg somit kaum ans Ziel führen: Die Subsumierbarkeit des Alterns unter einen bestimmten - sei es naturalistischen oder normativistischen - Krankheitsbegriff gibt keinen Aufschluss über den Umfang solidarisch zu tragender Leistungen. Zwar bringt der naturalistische Ansatz die zutreffende Intuition zum Tragen, dass sich ein tragfähiges Kriterium der Umfangsbegrenzung nicht auf subjektive Präferenzen zurückziehen darf, sondern intersubjektiv anerkennungsfähige Prinzipien geltend machen muss. Mit seiner naturalistischen Deutungsperspektive verfehlt er aber den genuin normativen Charakter dieser Prinzipien bereits im Ansatz und erweist sich so gerade wegen der prätendierten Wertfreiheit, die doch seine Objektivität und Zustimmungsfähigkeit verbürgen soll, im Kontext der Umfangsbestimmung solidarischer Gesundheitsversorgung als unbrauchbar. Hier tragen normativistische Konzeptionen insofern weiter, als sie auf den konstitutiven Wertbezug der zur Entscheidung stehenden Fragen verweisen. Allerdings können die Werte, auf die sie sich dabei beziehen, nicht ohne Weiteres theoretisch unterstellt werden. Sie sind von den Beteiligten und Betroffenen selbst aufzunehmen, ohne dass sich aus deren faktischen Stellungnahmen unmittelbar normative Direktiven ableiten ließen. 


\section{Ausblick: Jenseits von Krankheitsbehandlung und Wunscherfüllung}

Am Ende des eingeschlagenen Wegs zur Entscheidung über den Umgang mit der AntiAging-Medizin steht Ernüchterung. Schon der für die Entscheidung grundlegende Begriff der Krankheit ist nicht eindeutig definiert. Ähnliches gilt für den Begriff des Alterns und die Frage, in welcher Beziehung er zum Krankheitsbegriff steht. Und zuletzt zeigt sich, dass auch wenn die begrifflichen Schwierigkeiten auszuräumen wären, nur neue Hindernisse auf argumentativer Ebene aufträten. Insofern führt der besagte Entscheidungsweg nicht nur nicht zum angestrebten Ziel. Er endet vielmehr in prinzipieller Skepsis bezüglich des Versuchs, die Frage des Leistungsumfangs allein auf der Grundlage des Krankheitsbegriffs zu beantworten.

Angesichts solcher Schwierigkeiten wurde verschiedentlich vorgeschlagen, komplett auf den Krankheitsbegriff zu verzichten, da er praktisch entbehrlich sei und den Blick auf die entscheidenden Fragen eher verstelle [39]. Stattdessen sei der praktische „Regelungszweck“ ins Auge zu fassen, ,der dem Krankheitsbegriff im Recht der gesetzlichen Krankenversicherung zukommt“. Es ist demnach zu fragen, ,worin die normative Relevanz des Krankheitsbegriffs besteht“" ([38], S. 404): Warum soll gerade denjenigen Personen, die wir als krank bezeichnen, ein Anspruch auf medizinische Versorgung zukommen? Welche moralischen Intentionen verbinden wir mit dieser Praxis? Auf diesem Weg lassen sich die normativen Prämissen explizieren, die beim sozialrechtlichen Gebrauch des Krankheitsbegriffs im Spiel sind, und so einer offenen Auseinandersetzung zugänglich machen.

Damit eröffnet sich auch für die Anti-Aging-Medizin ein alternativer Entscheidungsweg, den sowohl ihre Befürworter als auch ihre Gegner akzeptieren könnten. Ihm zufolge ist die Frage der Kostenübernahme vom Krankheitsbegriff abzulösen und im Hinblick auf die Bewertung des Alterns und die Wünschbarkeit, Akzeptabilität oder Gebotenheit medizinischer Eingriffe in den Alterungsprozess zu erörtern [29]. Dabei ist zu bedenken, dass man sich in diesen Fragen nicht auf subjektive Präferenzen oder partikulare Welt- und Menschenbilder zurückziehen darf, sondern Entscheidungen zu treffen hat, die allgemein Anerkennung erlangen können. Aus diesem Grund sind einerseits die Auffassungen der Beteiligten und Betroffenen zur Kenntnis nehmen und in den Entscheidungsprozess einzubeziehen. Andererseits können aus ihren Wertvorstellungen nicht unmittelbar normative Folgerungen gezogen werden, weil die Legitimität normativer Überzeugungen nicht von ihrem faktischen Vorkommen, sondern von ihrer Verallgemeinerbarkeit abhängt. So sind Verfahren notwendig, die eine deliberativ erzielte, also durch Argumente qualifizierte Einigung ermöglichen.

Für die Gesundheitsversorgung im Allgemeinen hat Emanuel ein solches Verfahren entworfen, das auch als Muster für die Entscheidungsfindung im Bereich des Anti-Aging dienen könnte. Dabei ist der zentrale Gedanke, dass die Beteiligten sich nach fairen prozeduralen Regeln auf eine gemeinschaftliche gesundheitsbezogene Vorstellung guten Lebens verständigen, die der Bestimmung von Umfang, Prioritäten und einschränkenden Bedingungen solidarischer Gesundheitsversorgung zu Grunde zu legen ist ([16], S. 178). Natürlich hat dieser prozeduralistische Ansatz ebenfalls seine Schwierigkeiten [26] und bedürfte hinsichtlich des Anti-Aging einiger Spezifikationen [35]. Er hätte aber den entscheidenden Vorzug, die normativen Implikationen der Fragestellung weder naturalistisch kaschieren noch bloß faktischen Prozessen strategischer Aushandlung oder gesellschaftlichen Wertewandels überlassen zu müssen, sondern sie explizit als normative ansprechen und einer offenen Auseinandersetzung zugänglich machen zu können [36].

So deutet viel darauf hin, dass man im Blick auf die Anti-Aging-Medizin nicht zu tragfähigen Lösungen gelangen kann, ohne sich auf die in pluralistischen Gesellschaften stets 
kontroverse Diskussion von Vorstellungen guten Lebens einzulassen, in deren Horizont das Altern gedeutet und alternsbezogene Maßnahmen bewertet werden können. Während solche Vorstellungen im öffentlichen und akademischen Diskurs ihren Ort und ihre Berechtigung haben, kann der moderne demokratische Rechtsstaat sie bei der Begründung allgemein bindender politischer Regelungen freilich nicht mehr voraussetzen, sondern allenfalls als Ergebnis eines Verständigungsprozesses anstreben. Ein bestimmtes Verständnis des Alterns und seiner Bedeutung für das Leben im Ganzen kann nicht den Ausgangspunkt für Entscheidungen über Anti-Aging-Medizin im Rahmen öffentlicher Gesundheitsversorgung bilden, sondern höchstens das Ziel einer gesellschaftlichen Auseinandersetzung darüber, wie wir alt werden wollen und welche Rolle die Medizin dabei spielen soll.

Danksagung Die Arbeit an diesem Beitrag wurde unterstützt durch das Bundesministerium für Bildung und Forschung (BMBF) im Rahmen der Forschungskooperation $\mathrm{Zu}$ den ethischen Grenzen einer präferenzorientierten Medizin. Eine interdisziplinäre Analyse am Beispiel der Anti-Aging-Medizin, Teilprojekt 5: Die Verteilung knapper Gesundheitsressourcen zwischen Krankheitsorientierung und Präferenzerfüllung: gerechtigkeitsethische, ökonomische und gesundheitssystemische Implikationen der Anti-Aging-Medizin (Förderkennzeichen 01GP0622).

Interessenkonflikt Die Autoren geben an, dass keine Interessenkonflikte bestehen.

Open Access Dieser Artikel unterliegt den Bedingungen der Creative Commons Attribution Noncommercial License. Dadurch sind die nichtkommerzielle Nutzung, Verteilung und Reproduktion erlaubt, sofern der/die Originalautor/en und die Quelle angegeben sind.

\section{Literatur}

1. Amrhein L, Backes G (2007) Alter(n)sbilder und Diskurse des Alter(n)s. Anmerkungen zum Stand der Forschung. Z Gerontol Geriatr 40:104-111

2. Baltes D (2009) Der Wert der Fragilität. Überlegungen zum Stellenwert von Kontingenzargumenten im Rahmen der Enhancementdebatte. Z Med Ethik 55:351-369

3. BCCresearch (2009) Anti-aging products and services: the global market. http://www.bccresearch.com/ report/HLC060A.html. Zugegriffen: 20. Dez. 2010

4. Bobbert M (2000) Die Problematik des Krankheitsbegriffs und der Entwurf eines moralisch informativen Krankheitsbegriffs im Anschluss an die Moralphilosophie von Alan Gewirth. Ethica 8:405-440

5. Boorse C (1977) Health as a theoretical concept. Philos Sci 44:542-573

6. Bostrom N (2005) The fable of the dragon-tyrant. J Med Ethics 31:273-277

7. Bostrom N (2009) In defence of posthuman dignity. Bioethics 19:202-214

8. Blumenthal HT (2003) The aging-disease dichotomy: true or false? J Gerontol 58:138-145

9. Bruder J, Lucke C, Schramm A, Tews HP, Werner H (2005) Was ist Geriatrie? Expertenkommission der Deutschen Gesellschaft für Geriatrie und Deutschen Gesellschaft für Gerontologie zur Definition des Faches Geriatrie. http://afgib.de/Service_Downloads/Was_ist_Geriatrie.pdf.

10. Callahan D (1987) Setting limits. Medical goals in an aging society. Schuster, New York

11. Caplan AL (2005) Death as an unnatural process. EMBO Rep 6:72-75

12. Caplan AL, McCartney JJ, Sisti DA (Hrsg) (2004) Health, disease, and illness. Concepts in medicine. Georgetown University Press, Washington

13. Conrad P (2007) The medicalization of society. On the transformation of human conditions into treatable disorders. Johns Hopkins Press, Baltimore

14. Eberbach WH (2009) Möglichkeiten und rechtliche Beurteilung der Verbesserung des Menschen - Ein Überblick. In: Wienke A, Eberbach W, Kramer H-J, Janke K (Hrsg) Die Verbesserung des Menschen: tatsächliche und rechtliche Aspekte der wunscherfüllenden Medizin. Springer, Berlin, S 1-39

15. Ehni HJ, Marckmann G (2009) Social justice, health inequities, and access to new age-related interventions. Med Stud 1:281-295

16. Emanuel E (1991) The ends of human life. Medical ethics in a liberal polity. Havard University Press, Cambridge 
17. Evans JG (1988) Aging and disease. In: Evered D, Whalen J (Hrsg) Research and the aging population. Wiley, Chichester, S 38-57

18. Fedoryka K (1997) Health as a normative concept: towards a new conceptual framework. J Med Philos 22:143-160

19. Grey A de (2005) Life extension, human rights, and the rational refinement of repugnance. J Med Ethics 31:659-663

20. Izaks GJ, Westendorp RGJ (2003) Ill or just old? Towards a conceptual framework of the relation between ageing and disease. BMC Geriatr 3:7

21. Kass L (2001) L'Chaim and its limits. Why not Immortality? First Things 113:17-24

22. Kliemt H (2003) Rationierung im pluralen Rechtsstaat. In: Marckmann G (Hrsg) Gesundheitsversorgung im Alter. Zwischen ethischer Verpflichtung und ökonomischem Zwang. Schattauer, Stuttgart, S $59-71$

23. Lachs J (2004) Is aging a disease? HEC Forum 16:173-182

24. Mackey T (2003) An ethical assessment of anti-aging-medicine. J Anti-Aging Med 6:187-204

25. Maio G (2006) Die Präferenzorientierung der modernen Medizin als ethisches Problem. Ein Aufriss am Beispiel der Anti-Aging-Medizin. Z Med Ethik 52:339-354

26. Marckmann G (2002) Prioritäten im Gesundheitswesen: Zwischen Gerechtigkeit und gutem Leben. In: Brand A, Engelhardt D v, Simon A, Wehkamp KH (Hrsg) Individuelle Gesundheit versus Public Health? Lit-Verlag, Münster, S 178-189

27. Mazal W (1992) Krankheitsbegriff und Risikobegrenzung: Eine Untersuchung zum Leistungsrecht der gesetzlichen Krankenversicherung. Braumüller, Wien

28. Mazouz N, Werner MH, Wiesing U (Hrsg) (2004) Krankheitsbegriff und Mittelverteilung. Nomos, Baden-Baden

29. Murphy TF (1986) A cure for aging? J Med Philos 11:237-255

30. Nordenfeldt L (1987) On the nature of health: an action-theoretic approach. Kluwer, Dorderecht Boston

31. Olshansky SJ, Perry D, Miller RA, Butler RN (2006) The longevity dividend. Scientist 20:28-36

32. Rentsch Th (1995) Altern als Werden zu sich selbst. Philosophische Ethik der späten Lebenszeit. In: Borscheid P (Hrsg) Alter und Gesellschaft. Hirzel, Stuttgart, S 53-62

33. Schmorrte S (1990) Alter und Medizin. Die Anfänge der Geriatrie in Deutschland. Arch Sozialgesch 30:15-41

34. Schramme Th (2009) Ist Altern eine Krankheit? In: Knell A, Weber M (Hrsg) Länger Leben? Philosophische und biowissenschaftliche Perspektiven. Suhrkamp, Frankfurt a. M., S 235-263

35. Schweda M, Herrmann B, Marckmann G (2011) Anti-Aging Medizin in der gesetzlichen Krankenversicherung? Sozialrechtliche Entscheidungspraxis und gerechtigkeitsethische Reflexion. In: Maio G (Hrsg) Altwerden ohne alt zu sein? Ethische Grenzen der Anti-Aging-Medizin. Alber, Freiburg i. Br., S $172-193$

36. Werner MH (2001) Ethische Implikationen der Grenzziehung zwischen Gesundheit und Krankheit. Ethik Unterr 4:11-15

37. Werner MH (2002) Die Eingrenzung des Leistungsspektrums des solidarfinanzierten Gesundheitssystems als Herausforderung liberaler Konzeptionen politischer Ethik. Z Med Ethik 48:125-135

38. Werner MH, Wiesing U (2002) Lehren aus dem Fall Viagra? Der Krankheitsbegriff im Sozialrecht am Beispiel der erektilen Dysfunktion. Gesundheitswes 64:398-404

39. Wiesing U (1998) Kann die Medizin als praktische Wissenschaft auf eine allgemeine Definition von Krankheit verzichten? Z Med Ethik 44:83-97 